\title{
Fuzzy Linear Labeling
}

\author{
T. K. Mathew Varkey ${ }^{1}$ and T. D. Sreena ${ }^{2}$ \\ ${ }^{1}$ Department of Mathematics, T.K.M College of Engineering, Kollam, Kerala, India \\ ${ }^{2}$ Department of Mathematics, Sree Narayana College, Thrissur, Kerala, India \\ E-Mail:mathewvarkeytk@gmail.com, sreenatd100@gmail.com
}

\begin{abstract}
A fuzzy linear labeling of a graph is the existence of two injections which assigns values in [0,1]. This labeling fuzzifies the crisp graph. In this paper we show the admissibility of fuzzy linear labeling in some path related graphs.
\end{abstract}

Keywords: Path Graph, Square Graph, Duplicate Graph, HGraph, $\mathbf{P}_{\mathbf{n}} \odot K_{1}$.

AMS: 2010-05C78

\section{INTRODUCTION}

All graphs we consider in this paper are all finite, simple and undirected. The first definition of fuzzy graph by Kaufmann was based on Zadeh's fuzzy relations. Rosenfield considered fuzzy relation on fuzzy sets and developed the structure of fuzzy graphs. After the work of Rosenfield, Yeh and Bang introduced various connectedness concepts of graphs and digraphs into fuzzy graphs. In [1], A. Nagoor Gani and D. Rajalaxmi (a) Subahashini introduced the concept of fuzzy labeling.

A graph is said to be a fuzzy labeling graph if a fuzzy labeling is defined on it. A fuzzy graph $\mathrm{G}=(\mathrm{V}, \mathrm{m}, \rho)$ is a non-empty set $\mathrm{V}$ together with a pair of functions, $\mathrm{m}: \mathrm{V}$ $\rightarrow[0,1]$ and $\rho: \mathrm{V} \times \mathrm{V} \rightarrow[0,1]$ such that for all $\mathrm{x}, \mathrm{y}$ in $\mathrm{V}$, $\rho(x, y) \leq m(x) \Lambda m(y)$, where $m$ is the fuzzy vertex set of $G$ and $\rho$ is the fuzzy edge set of $G$ respectively. In this paper we identify some path related graphs that admits fuzzy linear labeling and proves the admissibility.

\section{MAIN RESULTS}

\section{Definition 2.1}

Let $G=(V, E)$ be a crisp graph, where $V$ is the ordered set of vertices and $E$ is the set of ordered pair of edges. Then $G$ has a fuzzy linear labeling if there exist two injections $\mathrm{m}$ and $\rho$ such that $\mathrm{m}: \mathrm{V} \rightarrow[0,1]$ defined by $\mathrm{m}\left(\mathrm{v}_{\mathrm{i}}\right)=\frac{i}{N}$, where $\mathrm{V}=\left(v_{1}, v_{2, \ldots .,} v_{N}\right), v_{i} \in V$ and

$\rho: \mathrm{E} \rightarrow[0,1]$ defined by

$$
\rho\left(v_{i}, v_{j}\right)=\frac{2 \mathrm{~m}\left(\mathrm{v}_{\mathrm{i}}\right)+\mathrm{m}\left(\mathrm{v}_{\mathrm{j}}\right)}{N}, 1 \leq i, j \leq N
$$

The graph $G$ which admits fuzzy linear labeling is called a fuzzy linear labeled graph.

\section{Example 2.2}

Consider the path graph $\mathrm{P}_{5}$, where $|V(G)|=5,|E(G)|=4$.

Let $\mathrm{V}=\left(\mathrm{v}_{1}, \mathrm{v}_{2}, \ldots ., \mathrm{v}_{5}\right)$ and

Define two functions $\mathrm{m}: \mathrm{V} \rightarrow[0,1]$ and $\rho: \mathrm{E} \rightarrow[0,1]$ as follows:

$$
\begin{aligned}
& \mathrm{m}\left(\mathrm{v}_{\mathrm{i}}\right)=\frac{i}{5}, 1 \leq i \leq 5 \text { and } \rho\left(\mathrm{v}_{\mathrm{i}}, \mathrm{v}_{\mathrm{i}+1}\right)=\frac{2 \mathrm{~m}\left(\mathrm{v}_{\mathrm{i}}\right)+\mathrm{m}\left(\mathrm{v}_{\mathrm{i}+1}\right)}{N}= \\
& \frac{3 i+1}{5^{3}}, \quad 1 \leq i \leq 4
\end{aligned}
$$

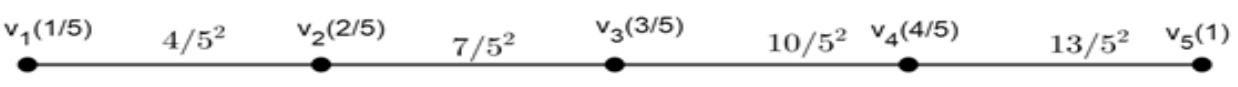

Fig. 1Fuzzy Linear Labeled Graph

\section{Theorem 2.3}

Every fuzzy linear labeled graph is a fuzzy graph.

\section{Proof}

From the definition of fuzzy linear labeling,

$\rho\left(v_{i}, v_{j}\right)=\frac{2 \mathrm{~m}\left(\mathrm{v}_{\mathrm{i}}\right)+\mathrm{m}\left(\mathrm{v}_{\mathrm{j}}\right)}{N} \leq \mathrm{m}\left(\mathrm{v}_{\mathrm{i}}\right) \wedge \mathrm{m}\left(\mathrm{v}_{\mathrm{j}}\right)$ for all $\mathrm{i}$ and $\mathrm{j}$ with $\mathrm{i}<\mathrm{j}$.

\section{Theorem 2.4}

Fuzzy linear labeling is a fuzzy labeling if and only if $\mathrm{j} \not \equiv$ $i(\bmod \mathrm{N}-2)$ for all $\mathrm{i}, \mathrm{j}$ with $\mathrm{i}<\mathrm{j}$.

\section{Proof}

By definition, $\rho: E \rightarrow[0,1]$ is said to be fuzzy labeling $\operatorname{if} \rho\left(v_{i}, v_{j}\right)<\mathrm{m}\left(\mathrm{v}_{\mathrm{i}}\right) \wedge \mathrm{m}\left(\mathrm{v}_{\mathrm{j}}\right)$ for all $\mathrm{i}$ and $\mathrm{j}$.

If possible, suppose that $\rho\left(v_{i}, v_{j}\right)=\mathrm{m}\left(\mathrm{v}_{\mathrm{i}}\right) \wedge \mathrm{m}\left(\mathrm{v}_{\mathrm{j}}\right)$ for some $\mathrm{i}$ and $\mathrm{j}$ such that $\mathrm{i}<\mathrm{j}$.

This means that $\rho\left(v_{i}, v_{j}\right)=\mathrm{m}\left(\mathrm{v}_{\mathrm{i}}\right) \Leftrightarrow \frac{2 \mathrm{~m}\left(\mathrm{v}_{\mathrm{i}}\right)+\mathrm{m}\left(\mathrm{v}_{\mathrm{j}}\right)}{N}=\mathrm{m}\left(\mathrm{v}_{\mathrm{i}}\right)$.

$\Leftrightarrow 2 \mathrm{i}+\mathrm{j}=\mathrm{i} \mathrm{N} \Leftrightarrow \mathrm{j} \not \equiv i(\bmod N-2)$.

\section{Result 2.5}

Two fuzzy linear labeled graphs are said to be equal if their underlying graphs are isomorphic. 


\section{Note 2.6}

For a fixed number of vertices, fuzzy linear labeled graph is unique.

\section{Definition 2.7}

A vertex $\mathrm{v}$ is said to be a common vertex in two fuzzy linear labeled graphs $\mathrm{G}_{1}$ and $\mathrm{G}_{2}$ if

$\mathrm{G}_{1}$ and $\mathrm{G}_{2}$ have the same number of vertices and $\mathrm{m}_{1}(\mathrm{v})=$ $\mathrm{m}_{2}(\mathrm{v})$.

An edge e is said to be a common edge of two fuzzy linear labeled graphs $G_{1}$ and $G_{2}$ if $G_{1}$ and $G_{2}$ have the same number of vertices and $\rho_{1}(\mathrm{e})=\rho_{2}(\mathrm{e})$.

\section{Result 2.8}

The order of a fuzzy linear labeled graph with $\mathrm{N}$ vertices $(\mathrm{N}+1) / 2$.

Proof

Order $=\sum_{i=1}^{N} m\left(v_{i}\right)=(1+2+\ldots+\mathrm{N}) / \mathrm{N}=(\mathrm{N}+1) / 2$.

\section{ON PATH RELATED GRAPHS}

In this section we show that the square graph of path, duplicate graph of path,H-graph of path and $\mathrm{P}_{\mathrm{n}} \odot K_{1}$ admit fuzzy linear labeling.

\section{Theorem 3.1}

The square of path $\mathrm{P}_{\mathrm{n}}^{2}$ admits fuzzy linear labeling.

Proof

Consider the square of $\mathrm{P}_{\mathrm{n}}, \mathrm{P}_{\mathrm{n}}{ }^{2}=(\mathrm{V}, \mathrm{E})$ where $|V|=\mathrm{n}$ and $|E|=2 \mathrm{n}-3$

Let $\mathrm{V}=\left(\mathrm{v}_{1}, \mathrm{v}_{2}, \ldots ., \mathrm{v}_{\mathrm{n}}\right)$.

Define two functions $\mathrm{m}: \mathrm{V} \rightarrow[0,1]$ and $\rho: \mathrm{E} \rightarrow[0,1]$ as follows:

$\mathrm{m}\left(\mathrm{v}_{\mathrm{i}}\right)=\frac{i}{n}, 1 \leq i \leq n$ and

$\rho: \mathrm{E} \rightarrow[0,1]$ as $\rho\left(\mathrm{v}_{\mathrm{i}}, \mathrm{v}_{\mathrm{i}+1}\right)=\frac{3 i+1}{n^{2}}, 1 \leq i \leq n-1$.

$$
\rho\left(\mathrm{v}_{\mathrm{i}}, \mathrm{v}_{\mathrm{i}+2}\right)=\frac{3 i+2}{n^{2}}, \quad 1 \leq i \leq n-2 .
$$

If possible, suppose that $\mathrm{m}$ is not injective.

Then there exist some $\mathrm{i}, \mathrm{j}$ such that $\mathrm{m}\left(\mathrm{v}_{\mathrm{i}}\right)=\mathrm{m}\left(\mathrm{v}_{\mathrm{j}}\right) \Longrightarrow$ $\frac{i}{n}=\frac{j}{n} \Rightarrow \mathrm{i}=\mathrm{j}$

Also if $\rho$ is not injective, then there exist some $i, j$ such that

1. $\rho\left(v_{i}, v_{i+1}\right)=\rho\left(v_{j}, v_{j+1}\right)$

$$
\Rightarrow \frac{3 i+1}{(2 n-1)^{2}}=\frac{3 j+1}{(2 n-1)^{2}} \Rightarrow \mathrm{i}=\mathrm{j} \text { for } 1 \leq i \leq n-1 \text {. }
$$

2. $\rho\left(v_{i}, v_{i+2}\right)=\rho\left(v_{j}, v_{j+2}\right)$

$$
\Rightarrow \frac{6 i+2}{(2 n-1)^{2}}=\frac{6 j+2}{(2 n-1)^{2}} \Rightarrow \mathrm{i}=\mathrm{j} \text { for } 1 \leq i \leq n-2 \text {. }
$$

3. $\rho\left(v_{\mathrm{i}}, \mathrm{v}_{\mathrm{i}+1}\right)=\rho\left(\mathrm{v}_{\mathrm{j}}, \mathrm{v}_{\mathrm{j}+2}\right)$

$\Rightarrow \frac{6 i+1}{(2 n-1)^{2}}=\frac{6 j+2}{(2 n-1)^{2}}$ for $1 \leq i \leq n-1$ and for

$1 \leq j \leq n-2 \Rightarrow 6(i-j)=1$, not possible since $\mathrm{i}$ and $j$ are natural numbers.

So $m$ and $\rho$ are injective.

\section{Theorem 3.2}

The duplicate graph of path graph $\mathrm{P}_{\mathrm{n}}$,admits fuzzy linear labeling.

Proof

Consider the duplicate graphDP $\mathrm{n}_{\mathrm{n}}=(\mathrm{V}, \mathrm{E})$ of $\mathrm{P}_{\mathrm{n}}$, where $|V|=$ $2 \mathrm{n},|E|=2 \mathrm{n}-2$.

Let $\mathrm{V}=\left(\mathrm{v}_{1}, \mathrm{v}_{2}, \ldots . \mathrm{v}_{\mathrm{n}}, \mathrm{u}_{1}, \mathrm{u}_{2}, \ldots \mathrm{u}_{\mathrm{n}}\right)$.

Define two functions $\mathrm{m}: \mathrm{V} \rightarrow[0,1]$ and $\rho: \mathrm{E} \rightarrow[0,1]$ as follows:

$\mathrm{m}\left(\mathrm{v}_{\mathrm{i}}\right)=\frac{i}{2 n}, \quad \mathrm{~m}\left(\mathrm{u}_{\mathrm{i}}\right)=\frac{i+n}{2 n}, 1 \leq i \leq n$ and

$\rho: \mathrm{E} \rightarrow[0,1]$ as $\rho\left(\mathrm{v}_{\mathrm{i}}, \mathrm{u}_{\mathrm{i}+1}\right)=\frac{3 i+n+1}{(2 n)^{2}}, \quad 1 \leq i \leq n-1$.

$\rho\left(\mathrm{v}_{\mathrm{i}}, \mathrm{u}_{\mathrm{i}-1}\right)=\frac{3 i-1+n}{(2 n)^{2}}, \quad 2 \leq i \leq n$.

If possible, suppose that $\mathrm{m}$ is not injective.

Then there exist some i,j such that

1. $\mathrm{m}\left(\mathrm{v}_{\mathrm{i}}\right)=\mathrm{m}\left(\mathrm{v}_{\mathrm{j}}\right) \Leftrightarrow \frac{i}{2 n}=\frac{j}{2 n} \Rightarrow \mathrm{i}=\mathrm{j}$.

2. $\mathrm{m}\left(\mathrm{u}_{\mathrm{i}}\right)=\mathrm{m}\left(\mathrm{u}_{\mathrm{j}}\right) \Leftrightarrow \frac{i+n}{2 n}=\frac{j+n}{2 n} \Rightarrow \mathrm{i}=\mathrm{j}$.

3. $\mathrm{m}\left(\mathrm{v}_{\mathrm{i}}\right)=\mathrm{m}\left(\mathrm{u}_{\mathrm{j}}\right) \Leftrightarrow \frac{i}{2 n}=\frac{j+n}{2 n} \Rightarrow \mathrm{i}-\mathrm{j}=\mathrm{n}$, not possible.

Also if $\rho$ is not injective, then there exist some $\mathrm{i}, \mathrm{j} \leq n$ such that

1. $\rho\left(\mathrm{v}_{\mathrm{i}}, \mathrm{u}_{\mathrm{i}+1}\right)=\rho\left(\mathrm{v}_{\mathrm{j}}, \mathrm{u}_{\mathrm{j}+1}\right)$ for $1 \leq i, j \leq n-1$

$\Rightarrow \frac{3 i+n}{(2 n)^{2}}=\frac{3 j+n}{(2 n)^{2}} \Rightarrow \mathrm{i}=\mathrm{j}$.

2. $\rho\left(\mathrm{v}_{\mathrm{i}}, \mathrm{u}_{\mathrm{i}-1}\right)=\rho\left(\mathrm{v}_{\mathrm{j}}, \mathrm{u}_{\mathrm{j}-1}\right)$ for $2 \leq i, j \leq n$

$\Rightarrow \frac{3 i-1+n}{(2 n)^{2}}=\frac{3 j-1+n}{(2 n)^{2}} \Rightarrow 3(i-j)=0 \Rightarrow \mathrm{i}=\mathrm{j}$.

3. $\rho\left(\mathrm{v}_{\mathrm{i}}, \mathrm{u}_{\mathrm{i}+1}\right)=\rho\left(\mathrm{v}_{\mathrm{j}}, \mathrm{u}_{\mathrm{j}-1}\right)$ for $1 \leq i \leq n-1$ and

$2 \leq j \leq n$

$\Rightarrow \frac{3 i+n}{(2 n)^{2}}=\frac{3 j-1+n}{(2 n)^{2}} \Rightarrow 3(i-j)=-1$, not possible.

So $m$ and $\rho$ are injective.

\section{Theorem 3.3}

The corona $\mathrm{P}_{\mathrm{n}} \odot K_{1}$ admits fuzzy linear labeling for $\mathrm{n} \not \equiv$ $1(\bmod 3)$.

Proof

Consider the corona $\mathrm{G}=\mathrm{P}_{\mathrm{n}} \odot K_{1}=(\mathrm{V}, \mathrm{E})$, where $|V|=$ 2n, $|E|=2 \mathrm{n}-1$.

Let $\mathrm{V}=\left(\mathrm{v}_{1}, \mathrm{v}_{2}, \ldots, \mathrm{v}_{\mathrm{n}}, \mathrm{u}_{1}, \mathrm{u}_{2}, \ldots, \mathrm{u}_{\mathrm{n}}\right)$.

Define $\mathrm{m}: \mathrm{V} \rightarrow[0,1]$ by $\mathrm{m}\left(\mathrm{v}_{\mathrm{i}}\right)=\frac{i}{2 n}, 1 \leq i \leq n$ and 
$\mathrm{m}\left(\mathrm{u}_{\mathrm{i}}\right)=\frac{i+n}{2 n} \quad, 1 \leq i \leq n$.

Define $\rho: \mathrm{E} \rightarrow[0,1]$ by $\rho\left(\mathrm{v}_{\mathrm{i}}, \mathrm{v}_{\mathrm{i}+1}\right)=\frac{3 i+1}{(2 n)^{2}}, \quad 1 \leq i \leq n-1$

$$
\rho\left(\mathrm{v}_{\mathrm{i}}, \mathrm{u}_{\mathrm{i}}\right)=\frac{3 i+n}{(2 n)^{2}}, \quad 1 \leq i \leq n .
$$

If possible, suppose that $m$ is not injective.

Then there exist some $i, j$ such that

1. $\mathrm{m}\left(\mathrm{v}_{\mathrm{i}}\right)=\mathrm{m}\left(\mathrm{v}_{\mathrm{j}}\right) \Rightarrow \frac{i}{2 n}=\frac{j}{2 n} \Rightarrow \mathrm{i}=\mathrm{j}$.

2. $\mathrm{m}\left(\mathrm{u}_{\mathrm{i}}\right)=\mathrm{m}\left(\mathrm{u}_{\mathrm{j}}\right) \Rightarrow \frac{i+n}{2 n}=\frac{j+n}{2 n} \Rightarrow \mathrm{i}=\mathrm{j}$.

3. $\mathrm{m}\left(\mathrm{v}_{\mathrm{i}}\right)=\mathrm{m}\left(\mathrm{u}_{\mathrm{j}}\right) \Rightarrow \frac{i}{2 n}=\frac{j+n}{2 n} \Rightarrow \mathrm{i}-\mathrm{j}=\mathrm{n}$, not possible.

Also if $\rho$ is not injective, then there exist some $\mathrm{i}, \mathrm{j} \leq n$ such that

1. $\rho\left(\mathrm{v}_{\mathrm{i}}, \mathrm{v}_{\mathrm{i}+1}\right)=\rho\left(\mathrm{v}_{\mathrm{j}}, \mathrm{v}_{\mathrm{j}+1}\right)$ for $1 \leq i, j \leq n-1$

$$
\Rightarrow \frac{3 i+1}{(2 n)^{2}}=\frac{3 j+1}{(2 n)^{2}} \Rightarrow \mathrm{i}=\mathrm{j} \text {. }
$$

2. $\rho\left(\mathrm{v}_{\mathrm{i}}, \mathrm{u}_{\mathrm{i}}\right)=\rho\left(\mathrm{v}_{\mathrm{j}}, \mathrm{u}_{\mathrm{j}}\right)$ for $1 \leq i, j \leq n$

$$
\Rightarrow \frac{3 i+n}{(2 n)^{2}}=\frac{3 j+n}{(2 n)^{2}} \Rightarrow 3(i-j)=0 \Rightarrow \mathrm{i}=\mathrm{j} \text {. }
$$

3. $\rho\left(\mathrm{v}_{\mathrm{i}}, \mathrm{v}_{\mathrm{i}+1}\right)=\rho\left(\mathrm{v}_{\mathrm{j}}, \mathrm{u}_{\mathrm{j}}\right)$ for $1 \leq i \leq n-1$ and $1 \leq j \leq n$ $\Rightarrow \frac{3 i+1}{(2 n)^{2}}=\frac{3 j+n}{(2 n)^{2}} \Longrightarrow 3(i-j)=\mathrm{n}-1 \Rightarrow n \equiv 1(\bmod 3)$

So $m$ and $\rho$ are injective.

\section{Theorem 3.4}

The H-graph of a path $\mathrm{P}_{\mathrm{n}}$ admits fuzzy linear labeling if $5 n \not \equiv 0(\bmod 6)$ and $n \not \equiv 1(\bmod 6)$

\section{Proof}

Consider the H-graph $\mathrm{G}=(\mathrm{V}, \mathrm{E})$ of path graphP $\mathrm{n}_{\mathrm{n}}$, where $|V(G)|=2 \mathrm{n}$,

$|E(G)|=2 \mathrm{n}-1$.

Let $\mathrm{V}=\left(\mathrm{v}_{1}, \mathrm{v}_{2}, \ldots ., \mathrm{v}_{\mathrm{n}}, \mathrm{u}_{1}, \mathrm{u}_{2}, \ldots, \mathrm{u}_{\mathrm{n}}\right)$.

Define $\mathrm{m}: \mathrm{V} \rightarrow[0,1]$ by $\mathrm{m}\left(\mathrm{v}_{\mathrm{i}}\right)=\frac{i}{(2 n)}, 1 \leq i \leq n \quad$ and $\mathrm{m}\left(\mathrm{u}_{\mathrm{i}}\right)$ $=\frac{i+n}{(2 n)}, 1 \leq i \leq n$

\section{Case (a): When $n$ is Even}

Without lossof generality assume that $\left(v_{\frac{n}{2}}, v_{\frac{n+2}{2}}\right)$ is an edge.

Define $\rho: \mathrm{E} \rightarrow[0,1]$ by $\rho\left(\mathrm{v}_{\mathrm{i}}, \mathrm{v}_{\mathrm{i}+1}\right)=\frac{3 i+1}{(2 n)^{2}}, \quad 1 \leq i \leq n-1$.

$\rho\left(\mathrm{u}_{\mathrm{i}}, \mathrm{u}_{\mathrm{i}+1}\right)=\frac{3 i+3 n+1}{(2 n)^{2}}, 1 \leq i \leq n-1$.

$\rho\left(v_{\frac{n}{2}}, u_{\frac{n}{2}+1}\right)=\frac{5 n+2}{2(2 n)^{2}}$.

If possible, suppose that $\mathrm{m}$ is not injective.

Then there exist some $i, j$ such that

1. $\mathrm{m}\left(\mathrm{v}_{\mathrm{i}}\right)=\mathrm{m}\left(\mathrm{v}_{\mathrm{j}}\right) \Rightarrow \frac{i}{2 n}=\frac{j}{2 n} \Rightarrow \mathrm{i}=\mathrm{j}$.

2. $\mathrm{m}\left(\mathrm{u}_{\mathrm{i}}\right)=\mathrm{m}\left(\mathrm{u}_{\mathrm{j}}\right) \Rightarrow \frac{i+n}{2 n}=\frac{j+n}{2 n} \Rightarrow \mathrm{i}=\mathrm{j}$.

3. $\mathrm{m}\left(\mathrm{v}_{\mathrm{i}}\right)=\mathrm{m}\left(\mathrm{u}_{\mathrm{j}}\right) \Rightarrow \frac{i}{2 n}=\frac{j+n}{2 n} \Rightarrow \mathrm{i}-\mathrm{j}=\mathrm{n}$, not possible.
Also if $\rho$ is not injective, then there exist some $\mathrm{i}, \mathrm{j} \leq n$ such that

1. $\rho\left(\mathrm{v}_{\mathrm{i}}, \mathrm{v}_{\mathrm{i}+1}\right)=\rho\left(\mathrm{v}_{\mathrm{j}}, \mathrm{v}_{\mathrm{j}+1}\right)$ for $1 \leq i, j \leq n-1$

$$
\Longrightarrow \frac{3 i+1}{(2 n)^{2}}=\frac{3 j+1}{(2 n)^{2}} \Longrightarrow \mathrm{i}=\mathrm{j} \text {. }
$$

2. $\rho\left(\mathrm{u}_{\mathrm{i}}, \mathrm{u}_{\mathrm{i}+1}\right)=\rho\left(\mathrm{u}_{\mathrm{j}}, \mathrm{u}_{\mathrm{j}+1}\right)$ for $1 \leq i, j \leq n-1$. $\Rightarrow \frac{3 i+3 n+1}{(2 n)^{2}}=\frac{3 j+3 n+1}{(2 n)^{2}} \Rightarrow 3(i-j)=0 \Rightarrow \mathrm{i}=\mathrm{j}$.

3. $\rho\left(\mathrm{v}_{\mathrm{i}}, \mathrm{v}_{\mathrm{i}+1}\right)=\rho\left(\mathrm{u}_{\mathrm{j}}, \mathrm{u}_{\mathrm{j}+1}\right)$ for $1 \leq i, j \leq n-1$ $\Longrightarrow \frac{3 i+1}{(2 n)^{2}}=\frac{3 j+3 n+1}{(2 n)^{2}} \Rightarrow 3(i-j)=3 \mathrm{n} \Rightarrow n=i-j$, not possible.

4. $\rho\left(v_{i}, v_{i+1}\right)=\rho\left(v_{\frac{n}{2}}, u_{\frac{n}{2}+1}\right) \Rightarrow \frac{3 i+1}{(2 n)^{2}}=\frac{5 n+2}{2(2 n)^{2}} \Rightarrow 6 \mathrm{i}+2=5 \mathrm{n}$ $+2$

$\Longrightarrow 5 \mathrm{n}=6 \mathrm{i} \Rightarrow 5 \mathrm{n} \equiv 0(\bmod 6)$.

5. $\rho\left(u_{i}, u_{i+1}\right)=\rho\left(v_{\frac{n}{2}}, u_{\frac{n}{2}+1}\right) \Rightarrow \frac{3 i+3 n+1}{(2 n)^{2}}=\frac{5 n+2}{2(2 n)^{2}} \Rightarrow$ $6 \mathrm{i}+6 \mathrm{n}+2=5 \mathrm{n}+2$

$\Rightarrow \mathrm{n}=-6 \mathrm{i}<0$, not possible.

So $\mathrm{m}$ and $\rho$ are injective.

\section{Case (b): When $n$ is Odd}

Without loss of generality assume that $\left(\frac{v_{n+1}}{2}, \frac{v_{n+1}}{2}\right)$ is an edge.

Define $\rho: \mathrm{E} \rightarrow[0,1]$ by $\rho\left(\mathrm{v}_{\mathrm{i}}, \mathrm{v}_{\mathrm{i}+1}\right)=\frac{3 i+1}{(2 n)^{2}}, \quad 1 \leq i \leq n-1$.

$\rho\left(\mathrm{u}_{\mathrm{i}}, \mathrm{u}_{\mathrm{i}+1}\right)=\frac{3 i+3 n+1}{(2 n)^{2}}, 1 \leq i \leq n-1$.

$\rho\left(v_{\frac{n+1}{2}}, u_{\frac{n+1}{2}}\right)=\frac{5 n+3}{2(2 n)^{2}}$.

If possible, suppose that $\mathrm{m}$ is not injective.

Then there exist some $\mathrm{i}, \mathrm{j}$ such that

1. $\mathrm{m}\left(\mathrm{v}_{\mathrm{i}}\right)=\mathrm{m}\left(\mathrm{v}_{\mathrm{j}}\right) \Rightarrow \frac{i}{2 n}=\frac{j}{2 n} \Rightarrow \mathrm{i}=\mathrm{j}$.

2. $\mathrm{m}\left(\mathrm{u}_{\mathrm{i}}\right)=\mathrm{m}\left(\mathrm{u}_{\mathrm{j}}\right) \Rightarrow \frac{i+n}{2 n}=\frac{j+n}{2 n} \Rightarrow \mathrm{i}=\mathrm{j}$.

3. $\mathrm{m}\left(\mathrm{v}_{\mathrm{i}}\right)=\mathrm{m}\left(\mathrm{u}_{\mathrm{j}}\right) \Rightarrow \frac{i}{2 n}=\frac{j+n}{2 n} \Rightarrow \mathrm{i}-\mathrm{j}=\mathrm{n}$, not possible.

Also if $\rho$ is not injective, then there exist some $\mathrm{i}, \mathrm{j} \leq n$ such that

1. $\rho\left(\mathrm{v}_{\mathrm{i}}, \mathrm{v}_{\mathrm{i}+1}\right)=\rho\left(\mathrm{v}_{\mathrm{j}}, \mathrm{v}_{\mathrm{j}+1}\right)$ for $1 \leq i, j \leq n-1$ $\Rightarrow \frac{3 i+1}{(2 n)^{2}}=\frac{3 j+1}{(2 n)^{2}} \Rightarrow \mathrm{i}=\mathrm{j}$.

2. $\rho\left(\mathrm{u}_{\mathrm{i}}, \mathrm{u}_{\mathrm{i}+1}\right)=\rho\left(\mathrm{u}_{\mathrm{j}}, \mathrm{u}_{\mathrm{j}+1}\right)$ for $1 \leq i, j \leq n-1$. $\Rightarrow \frac{3 i+3 n+1}{(2 n)^{2}}=\frac{3 j+3 n+1}{(2 n)^{2}} \Rightarrow 3(i-j)=0 \Longrightarrow \mathrm{i}=\mathrm{j}$.

3. $\rho\left(\mathrm{v}_{\mathrm{i}}, \mathrm{v}_{\mathrm{i}+1}\right)=\rho\left(\mathrm{u}_{\mathrm{j}}, \mathrm{u}_{\mathrm{j}+1}\right)$ for $1 \leq i, j \leq n-1$ $\Rightarrow \frac{3 i+1}{(2 n)^{2}}=\frac{3 j+3 n+1}{(2 n)^{2}} \Rightarrow 3(i-j)=3 \mathrm{n} \Rightarrow n=i-j$, not possible.

4. $\rho\left(v_{i}, v_{i+1}\right)=\rho\left(v_{\frac{n+1}{2}}, u_{\frac{n+1}{2}}\right) \Rightarrow \frac{3 i+1}{(2 n)^{2}}=\frac{5 n+3}{2(2 n)^{2}} \Rightarrow 6 i+2=5 n$ $+3$ $\Rightarrow 5 \mathrm{n}+1=6 \mathrm{i} \Rightarrow \mathrm{n} \equiv 1(\bmod 6)$.

5. $\rho\left(u_{i}, u_{i+1}\right)=\rho\left(\frac{v_{n+1}}{2}, u_{\frac{n+1}{2}}\right) \Rightarrow \frac{3 i+3 n+1}{(2 n)^{2}}=\frac{5 n+3}{2(2 n)^{2}} \Rightarrow$ $6 \mathrm{i}+6 \mathrm{n}+2=5 \mathrm{n}+3$

$\Rightarrow \mathrm{n}=-6 \mathrm{i}+1<0$, not possible.

So $m$ and $\rho$ are injective. 


\section{CONCLUSION}

In this paper we introduced fuzzy linear labeling and some of its properties. We also discuss the admissibility of fuzzy linear labeling in some path related graphs. We show that we can fuzzify the crisp graphs using fuzzy linear labeling.

\section{REFERENCES}

[1] A. NagoorGani and D. Rajalaxmi (a) Subahashini, "Properties of Fuzzy Labeling Graph", Applied Mathematical Sciences, Vol. 6, No.70, pp. 3461-3466, 2012.

[2] Sunil Mathew and M. S. Sunitha, "Types of Arcs in Fuzzy Graphs", Information Sciences, Vol. 179, pp. 1760-176, 2009.
[3] T. Pathinathan, J. Jon Arockiaraj and J. Jesintha Rosline, "Hesistancy Fuzzy Graphs", Indian Journal of Science and Technology, Vol 8, No. 35, Dec. 2015.

[4] M. A. Rifayathali, A. Prasanna, S. Ismail Mohideen, "Hesistancy Fuzzy Magic Labeling Graph", Springer.

[5] N. S. Hungund and D. G. Akka, "Super Edge -Magic Strength of Some families of Graphs", Bulletin of the Marathwada Mathematical Society, Vol. 12, No. 1, pp. 47-54, June 2011.

[6] George J. Klir and Bo Yuan, Fuzzy Sets and Fuzzy Logic - Theory and Applications, ${ }^{\text {st }}$ Edition, Prentice Hall, 1995.

[7] Sunil Mathew and M. S. Sunitha, Fuzzy Graphs- Basics, Concepts and Applications, Lap Lambert Academic Publishing, August 17, 2012.

[8] John N. Mordeson and Premchnand S. Nair, Fuzzy Graphs and Fuzzy Hypergraphs, Physica-Verlag, 2014. 